\title{
Neuroendocrine responses to traumatic brain injury
}

\author{
Fahrettin Kelestimur ${ }^{1}$ (1)
}

Published online: 29 April 2019

(c) Springer Science+Business Media, LLC, part of Springer Nature 2019

Traumatic brain injury (TBI) is a serious public health problem worldwide associated with increased mortality and disability especially among young people. Pituitary dysfunction was described for the first time a century ago occurring as a consequence of TBI [1]. Until 20 years ago, post-traumatic pituitary dysfunction received little attention, and during the following years, increasing numbers of papers were published in the literature. A special issue of Pituitary was devoted to TBI-induced hypopituitarism in 2005. Studies of neuroendocrine changes and brain injury have expanded the frontiers of endocrinology. The increasing amount of new data has required this current special issue on TBI-induced neuroendocrine changes. Scientists and clinicians from a variety of disciplines including endocrinology, neurology, pathology, immunology, stem cell, developmental biology and neurosurgery kindly contributed to this special issue of Pituitary.

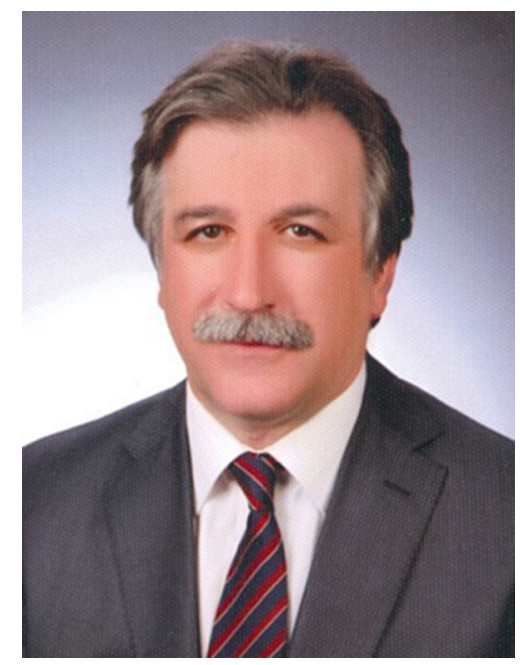

Fahrettin Kelestimur

fktimur@erciyes.edu.tr; fahrettin.kelestemur@yeditepe.edu.tr

1 Faculty of Medicine, Yeditepe University, Istanbul, Turkey
Benvenga reports historical perspectives on TBI-induced hypopituitarism in his inspiring paper. Tan and Hutchinson report on the neurosurgical approach to TBI-induced pituitary dysfunction. The frequency of TBI is extremely common but the prevalence of pituitary dysfunction following TBI is still a matter of debate. Glynn and Agha nicely report on the frequency of TBI-induced pituitary dysfunction and on the diagnostic assessment of pituitary functions in patients with TBI. Clinical and therapeutic aspects of TBI-induced pituitary dysfunction are presented by Caputo et al. They also discuss metabolic alterations in TBI-induced pituitary dysfunction. One of the disturbing consequences of TBI is cognitive impairment. Pavlovic et al. review the effects of TBI itself and TBI-induced pituitary dysfunction, GH deficiency in particular, on cognitive dysfunction. Neuroendocrine changes detected in the acute phase are reviewed by Ntali and Tsagarakis. Although rare, diabetes insipidus is another crucially important consequence of TBI, and an entirely updated paper on posterior pituitary dysfunction due to TBI is presented by by Tudor and Thompson. Karaca et al. draw attention to subarachnoid haemorrhage as a cause of hypopituitarism in their compelling review. Chronic repetitive head trauma is a newly discovered cause of hypopituitarism. Hacioglu et al. review pituitary dysfunction following sports-related TBI. Temizkan and Kelestimur summarize underlying mechanisms responsible for development of pituitary, and De Bellis et al. present the role of autoimmunity in development of post-traumatic hypopituitarism. An experienced team of neuropathologists, Sav et al. have assembled the published data regarding TBI associated histopathologic changes from 1918 through 2018, revealing a conundrum of accumulated histologic data to shed light in this challenging field. An experimental TBI model yields new insight into the mechanism and treatment of pituitary dysfunction. This seminal review by Vennekens and Vankelecom will stimulate basic scientists including stem cell researchers to develop new therapeutic approaches in treatment of hypopituitarism using pituitary stem cells.

I am honored to have served as guest editor for this special issue and I would like to give special thanks to Shlomo 
Melmed, Pituitary editor-in-chief, and also thank the authors for their scientific contribution and Springer editorial staff for their meticulous effort to efficiently publish this special issue. I hope that you will find this thematic issue interesting and useful by providing the most current knowledge and create awareness in the medical community. I wish you all a pleasant reading.

\section{Reference}

1. Cyran E (1918) Hypophysenschadigung durch schadelbasisfraktur. Dtsch Med Wochenschr 44(45): 1261

Publisher's Note Springer Nature remains neutral with regard to jurisdictional claims in published maps and institutional affiliations. 